2

\title{
Relative velocity measurement with spectrometer and its integrated navigation
}

\author{
Zhonghua Wang ${ }^{1}$, Yiming Peng ${ }^{2}$ and Jiu Liu ${ }^{3, *}$ \\ 1 School of Information and Engineering, Nanchang Hangkong University, Nanchang, 330063, People's \\ Republic of China; wangzhonghua@nchu.edu.cn \\ 2 School of Information and Engineering, Nanchang Hangkong University, Nanchang, 330063, People's \\ Republic of China; pengyiming1021@qq.com \\ 3 College of Information Science and Engineering, Wuhan University of Science and Technology, Wuhan \\ 430081, People's Republic of China; liujin@wust.edu.cn \\ * Correspondence: liujin@wust.edu.cn; Tel.: +8613971150792
}

\begin{abstract}
In order to enhance the accuracy of the relative velocity measurement for the Mars explorer formation flight, we develop a relative velocity measurement method. In this method, the spectrometers at two Mars explorers are adopted to measure the starlight frequency shift and to estimate the velocity with respect to the star. Unfortunately, the instantaneous velocity of star can not be predicted accurately, which results in a large error in the velocity measurement. The difference of these two velocities, which does not include the proper motion of star, is the relative velocity between a pair of Mars explorers at the direction of the star. However, this navigation method can not work alone because of unobservability. To make the navigation system observable and improve the accuracy of both absolute and relative navigation for the Mars explorer formation flight, we combine it with X-ray pulsar navigation and the inter-satellite links, and propose an autonomous integrated navigation method with observability. In this integrated navigation scheme, the extended Kalman filter is adopted to deal with the relative velocity, the inter-satellite links and the pulse time-of-arrival, and estimate the absolute and relative navigation information for the Mars explorer formation flight. The simulation results demonstrate that both absolute and relative navigation accuracy of the proposed method are higher than that of the pulsar navigation, especially the relative one.
\end{abstract}

Keywords: Navigation; Velocity measurement; X-ray pulsar; Mars explorer; Formation flight

\section{Introduction}

The formation flight of the deep space explorers is a novel area of aerospace technique [1,2]. The formation flight can increase redundancy backup, decentralize the risk, and provide a multi-platform for deep space exploration [3]. In the area of the deep space exploration, the navigation accuracy for the transfer orbit is very important. Traditional ground station-based navigation method can not provide high-accuracy and real-time navigation information. Consequently, the autonomous navigation systems [4,5], which need not communicate with ground stations, are highly attractive. It is worth emphasizing that both absolute and relative navigation information of the Mars explorer formation flight are very important, and the relative navigation has a higher accuracy requirement. 
X-ray pulsar navigation [6] is a novel autonomous navigation method for deep space exploration, which uses the X-ray sensor at spacecraft to observe X-ray pulsar and get the pulse TOA (time-ofarrival) $[7,8]$. Using the pulse TOA as the measurement, the $X$-ray pulsar navigation system can provide absolute position information for Mars explorers. Naturally, it can also estimate the relative navigation information via calculating the difference of the absolute ones. Emadzadeh has proposed the relative navigation method between two deep space explorers using X-ray pulsar [9]. In this method, the pulse TOAs are used as measurements, and based on models of the spacecraft and IMU dynamics, a Kalman filter is adopted to obtain the relative position and velocity. The inter-satellite link [10] is a mature measurement for relative navigation, which can provide high-accuracy range between two spacecrafts. This technique has been applied to some missions. For example, GPS Block IIR and Block IIF satellites have fixed crosslink transponder. Xiong has proposed the relative navigation with the inter-satellite links and the time difference of arrival from X-ray pulsar[11]. Its relative navigation accuracy is enhanced. Combined with the orbit dynamic model, this method can also provide the absolute navigation information with the low earth orbit. However, it is not suitable to the transfer orbit, and its relative accuracy is limited. The vision-based relative navigation is a commonly-used method, which utilizes the star sensor to observe the flying Mars explorer according to the explorer reflectivity. However, it is subjected to the long distance between a pair of Mars explorers. Based this method, Xiong has also develop a relative navigation method based on the star sensors and inter-satellite links, where the star sensors are utilized to observe other satellites [10]. Thus, this method is still only fit for the short-distance between two spacecrafts.

As we know, currently, the celestial information on the star which can be measured includes the direction vector and the relative velocity. The traditional celestial navigation method adopts the direction of star as the measurement. And the relative velocity measurement can not be exploited now. In fact, the velocity measurement accuracy of the spectrometer is very high, and can reach $0.01 \mathrm{~m} / \mathrm{s}$. When we utilize the spectrometer to observe the light from the celestial body, the relative velocity between the celestial body and the spacecraft can be estimated according to the frequency shift of the light. However, currently, we are not able to high-accurately predict the instantaneous velocity of the celestial body, especially the star. That is to say, we can not estimate the absolute velocity of spacecraft with the starlight frequency shift.

In this paper, in order to exploit the starlight and resist the impact of the proper motion of star on the spacecraft velocity estimation, we develop a novel relative velocity estimation method with starlight. In this method, the velocity between the spacecraft and the celestial body is measured by the spectrometer at spacecraft. The difference of these two velocities of two spacecrafts is the relative velocity between two spacecrafts at the direction of star. However, we find that the relative velocity navigation system is not observable. In order to make the celestial autonomous navigation system for formation flight observable, we combine the relative velocity measurement method with the X-ray pulse TOA and the inter-satellite links, and propose a novel integrated navigation scheme, where the EKF (extended Kalman filter) is adopted as the navigation filter to provide the absolute and relative navigation information.

This paper is organized into five sections. After the introduction, the relative velocity measurement using starlight frequency shift is developed in Section 2. The integrated navigation filter is described in Section 3. The simulation results in Section 4 demonstrate the robustness and accuracy of this presented method and conclusions are drawn in Section 5.

\section{Relative Velocity Measurement Using Starlight Frequency Shift}

In the traditional celestial navigation method, the direction of the star is fully exploited. The star sensor is employed to measure the direction of star precisely. And the attitude of spacecraft can be determined accurately with three or more stars. When the spacecraft is near a celestial body such as the earth, the spacecraft's position can be also determined via measuring the star-earth angle or the 
elevation angle to star. But in the transfer orbit, the accuracy of this method is very low due to the long-distance between the spacecraft and the near celestial body.

In fact, both the star direction and the starlight frequency can be measured from the star. Currently, the relative velocity measurement can be obtained by the spectrometer precisely. In this section, we develop a novel idea where the navigation measurement is the frequency shift of starlight but not the direction of star.

\subsection{The relative velocity measurement}

Before designing the relative velocity measurement method, we introduce and analyse the absolute velocity measurement based on the starlight frequency shift. The details are as follows: in general, the frequency spectrum of starlight can be measured by the spectrometer placed at the spacecraft. Due to the motion of the spacecraft with respect to the light source (star), the spectral lines of starlight are shifted from the original positions corresponding to their wavelength, which is called the frequency shift. Conversely, the velocity of spacecraft with respect to the star can be solved from the starlight frequency shift. The direction vector of star, $\boldsymbol{s}$, may be considered constant throughout the solar system since the star is so distant from the Solar system. The velocity of spacecraft with respect to the star is the velocity at the direction of the star. This velocity measurement is highly accurate due to the high performance of the spectrometer, and its accuracy is less than $0.01 \mathrm{~m} / \mathrm{s}$. The velocity measurement model can be expressed as:

$$
v_{s}^{a}=\boldsymbol{s} \cdot \boldsymbol{v}-\boldsymbol{s} \cdot \boldsymbol{v}_{s}
$$

where $v_{s}^{a}$ is the measured velocity with respect to the star, $\boldsymbol{v}$ and $\boldsymbol{v}_{s}$ are the spacecraft and star velocities with respect to the SSB (solar system barycentre) respectively. From Eq.(1), it can be seen that when $v_{s}^{a}, \boldsymbol{s}$ and $\boldsymbol{v}_{s}$ are precise, the spacecraft velocity at the direction of $\operatorname{star}, \boldsymbol{s} \cdot \boldsymbol{v}$, can be estimated accurately. However, subjected to the current measurement technology and celestial observation data, a large error exists in the instantaneous radial velocity of the star, $\boldsymbol{s} \cdot \boldsymbol{v}_{s}$. Therefore, the velocity measurement can be represented as:

$$
v_{s}^{a}=\boldsymbol{s} \cdot \boldsymbol{v}-\left(\boldsymbol{s} \cdot \boldsymbol{v}_{s}+\Delta v\right)
$$

where $\Delta v$ is a large velocity error. From Eq.(2), we can seen that the estimated velocity at the direction of star is $\boldsymbol{s} \cdot \boldsymbol{v}+\Delta v$. Therefore, the velocity measurement method based on starlight frequency shift can not be applied to the absolute velocity measurement due to the large error in the radial velocity estimation of the star.

If the velocity measurement model does not include $\boldsymbol{v}_{s}, \boldsymbol{s} \cdot \boldsymbol{v}$ can be estimated precisely according to $v_{s}^{a}$. Based on this idea, we build the relative velocity measurement model for formation flight based on the starlight frequency shift, which does not include radial velocity of the star. The basic principle of this measurement method is presented in Figure 1. In this paper, the formation is composed by two Mars explorers. $\boldsymbol{v}_{0}$ and $\boldsymbol{v}_{1}$ are the velocities of Mars explorer 0 and 1 with respect to SSB, respectively. Correspondingly, $\boldsymbol{s} \cdot\left(\boldsymbol{v}_{0}-\boldsymbol{v}_{s}\right)$ and $\boldsymbol{s} \cdot\left(\boldsymbol{v}_{1}-\boldsymbol{v}_{s}\right)$ are the velocities of the Mars explorer 0 and 1 with respect to the star, respectively. These two velocity values are measured by the spectrometers placed at two Mars explorers respectively. According to Eq.(2), it can be seen that there is a large predicted error in the predicted radial velocity, $\boldsymbol{s} \cdot \boldsymbol{v}_{s}+\Delta v$. Thus, we can not obtain accurate $\boldsymbol{v}_{0}$ and $\boldsymbol{v}_{1}$ even if the measured velocities with respect to the star are precise.

Fortunately, we can obtain the relative velocity of two explorers at the direction of the star, $v_{s}^{r}$, as follows: 


$$
v_{s}^{r}=\boldsymbol{s} \cdot\left(\boldsymbol{v}_{0}-\boldsymbol{v}_{s}\right)-\boldsymbol{s} \cdot\left(\boldsymbol{v}_{1}-\boldsymbol{v}_{s}\right)=\boldsymbol{s} \cdot\left(\boldsymbol{v}_{0}-\boldsymbol{v}_{1}\right)
$$

128

129 130

From equation (3), it can be seen that $v_{s}^{r}$ is not subjected to the radial motion of the star. Namely, $v_{s}^{r}$ is not affected by the predicted velocity error of star. Therefore, we can get accurate $v_{s}^{r}$ form $\boldsymbol{s} \cdot\left(\boldsymbol{v}_{0}-\boldsymbol{v}_{s}\right)$ and $\boldsymbol{s} \cdot\left(\boldsymbol{v}_{1}-\boldsymbol{v}_{s}\right)$.

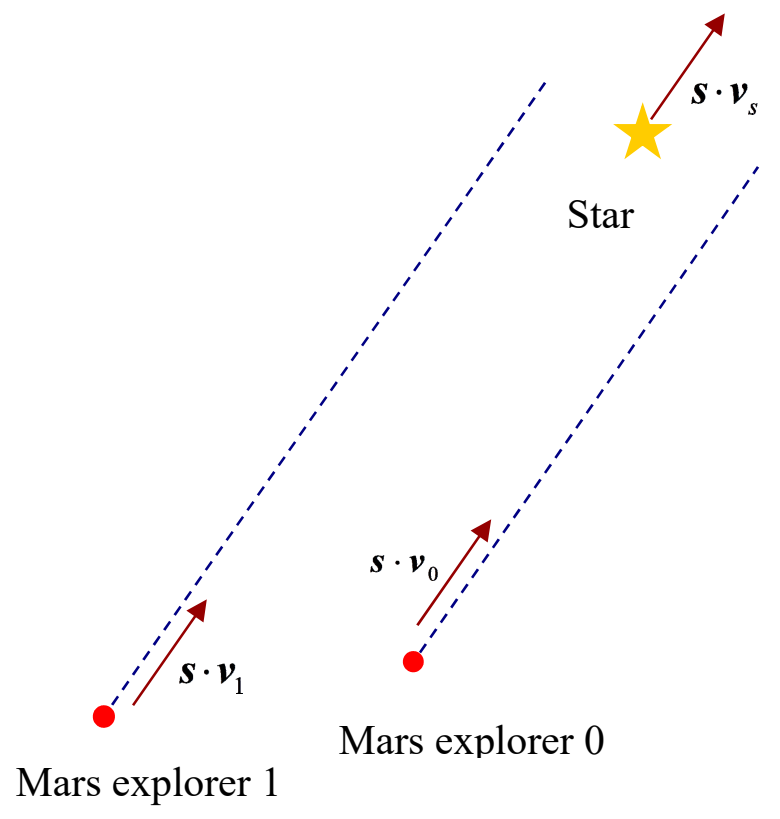

Figure 1. The basic principle of relative navigation based on starlight frequency shift

132

133

134
Next, we build the relative velocity measurement model. Suppose that $\omega_{v 0}^{l}$ and $\omega_{v 1}^{l}$ are the measurement noises caused by the spectrometers at the explorer 0 and 1 respectively. For the 1-th navigation star, the relative velocity, $v_{s l}^{r}$, can be represented as:

$$
v_{s l}^{r}=\left[\boldsymbol{s} \cdot\left(v_{0}-v_{s}\right)+\omega_{v 0}^{b}\right]-\left[s \cdot\left(v_{1}-v_{s}\right)+\omega_{v 1}^{b}\right]=s^{l} \cdot\left(v_{0}-v_{1}\right)+\omega_{v 0}^{b}-\omega_{v 1}^{b}
$$

where $l=1,2, \ldots, L$. L is the number of adopted navigation stars. $\boldsymbol{s}^{l}$ is the direction of the 1th navigation star.

For the navigation system with multiple navigation stars, the relative velocity measurement

$$
\boldsymbol{Y}^{s}(t)=h^{s}(\boldsymbol{X}, t)+\boldsymbol{\omega}_{v 0}-\boldsymbol{\omega}_{v 1}
$$

model can be represented as:

where the measurement vector, $\boldsymbol{Y}^{s}(t)$, the measurement equations $h^{s}(\boldsymbol{X}, t)$, and the noises, $\omega_{v 0}, \omega_{v 1}$ can be represented as:

$$
\boldsymbol{Y}^{s}(t)=\left[v_{s 1}^{r}, v_{s 2}^{r}, \cdots, v_{s L}^{r}\right]^{\mathrm{T}}
$$




$$
\begin{gathered}
h^{s}(\boldsymbol{X}, t)=\left[h_{1}^{s}(\boldsymbol{X}, t), h_{2}^{s}(\boldsymbol{X}, t), \cdots, h_{L}^{s}(\boldsymbol{X}, t)\right]^{\mathrm{T}} \\
h_{l}^{s}(\boldsymbol{X}, t)=\boldsymbol{s}^{l} \cdot\left(\boldsymbol{v}_{0}-\boldsymbol{v}_{1}\right) \\
\boldsymbol{\omega}_{v 0}=\left[\omega_{v 0}^{1}, \omega_{v 0}^{2}, \cdots, \omega_{v 0}^{L}\right]^{\mathrm{T}} \\
\boldsymbol{\omega}_{v 1}=\left[\omega_{v 1}^{1}, \omega_{v 1}^{2}, \cdots, \omega_{v 1}^{L}\right]^{\mathrm{T}}
\end{gathered}
$$

141

142

143

144

145

146

147

148

149

150

\subsection{The navigation performance analysis}

In this section, we analyze the performance of the relative navigation based on starlight frequency shift from the following three aspects: (1) the observability of navigation system; (2) the measurement noise; (3) the applicable conditions.

(1) The observability of the navigation system. The observability of the navigation system is an important evaluation index for navigation performance. Next, we construct the observability matrix and analyze it.

In the transfer orbit for the Mars exploration, the $\left|\boldsymbol{r}^{(i)}\right|$ is on the order of $1011 \mathrm{~m} . \partial \dot{\boldsymbol{v}}^{(i)} / \partial \boldsymbol{r}^{(i)}$ is on the order of $\mu_{s} /\left|\boldsymbol{r}^{(i)}\right|^{3}$, whose value is on the order of 10-13 and can be ignored.

The state transfer matrix $\boldsymbol{A}_{k}$ can be represented as:

$$
\boldsymbol{A}_{k}=\left.\frac{\partial f(\boldsymbol{X}, t)}{\partial \boldsymbol{X}}\right|_{\boldsymbol{X}=\hat{\boldsymbol{X}}_{k}} \approx\left[\begin{array}{l}
\boldsymbol{0}_{3 \times 3}, \boldsymbol{I}_{3 \times 3}, \boldsymbol{0}_{3 \times 3}, \boldsymbol{0}_{3 \times 3} \\
\boldsymbol{0}_{3 \times 3}, \boldsymbol{0}_{3 \times 3}, \boldsymbol{0}_{3 \times 3}, \boldsymbol{0}_{3 \times 3} \\
\boldsymbol{0}_{3 \times 3}, \boldsymbol{0}_{3 \times 3}, \boldsymbol{0}_{3 \times 3}, \boldsymbol{I}_{3 \times 3} \\
\boldsymbol{0}_{3 \times 3}, \boldsymbol{0}_{3 \times 3}, \boldsymbol{0}_{3 \times 3}, \boldsymbol{0}_{3 \times 3}
\end{array}\right]
$$

151 where $\partial f(\boldsymbol{X}, t)$ is the orbit dynamic model.

For the frequency shift-based navigation, the relative velocity measurement matrix, $\boldsymbol{H}_{k}$, can be 153 represented as follows:

$$
\boldsymbol{H}_{k}=\left.\frac{\partial h(\boldsymbol{X}, t)}{\partial \boldsymbol{X}}\right|_{\boldsymbol{X}=\hat{\boldsymbol{X}}_{k}}=\left[\boldsymbol{0}_{L \times 3}, \boldsymbol{S}_{L \times 3}, \boldsymbol{0}_{L \times 3},-\boldsymbol{S}_{L \times 3}\right]
$$

According to Eq.(11) and (12), we can construct the observability matrix, $\boldsymbol{O}$, which can be expressed as follows:

$$
\boldsymbol{O}=\left[\begin{array}{c}
\boldsymbol{H}_{k} \\
\boldsymbol{H}_{k} \boldsymbol{A}_{k} \\
\vdots \\
\boldsymbol{H}_{k}\left(\boldsymbol{A}_{k}\right)^{\mathrm{m}}
\end{array}\right]=\left[\begin{array}{c}
\boldsymbol{0}_{L \times 3}, \boldsymbol{S}_{L \times 3}, \boldsymbol{0}_{L \times 3},-\boldsymbol{S}_{L \times 3} \\
\boldsymbol{0}_{L \times 3}, \boldsymbol{0}_{L \times 3}, \boldsymbol{0}_{L \times 3}, \boldsymbol{0}_{L \times 3} \\
\vdots \\
\boldsymbol{0}_{L \times 3}, \boldsymbol{0}_{L \times 3}, \boldsymbol{0}_{L \times 3}, \boldsymbol{0}_{L \times 3}
\end{array}\right]
$$

The rank of the observability matrix is: 


$$
\operatorname{rank}(\boldsymbol{O})=\left\{\begin{array}{l}
L, L<3 \\
3, L \geq 3
\end{array}\right.
$$

When the observability matrix is a full column rank matrix, the navigation system is observable. Obviously, the matrix $\boldsymbol{O}$ is not a full column rank matrix. Thus, the starlight frequency shift-based navigation system is unobservable. Namely, this navigation method can not work alone, and is only utilized as an aided navigation method. In this paper, we combine it with the X-ray pulsar navigation system, which is observable.

(2) The measurement noise. Suppose that the variances of $\omega_{v 0}^{l}$ and $\omega_{v 1}^{l}$ are $R_{v}, \omega_{v 0}^{l}$ and $\omega_{v 1}^{l}$ are uncorrelated.

$$
\begin{aligned}
& E\left[\left(\omega_{v 0}^{l}\right)^{2}\right]=R_{v} \\
& E\left[\left(\omega_{v 1}^{l}\right)^{2}\right]=R_{v} \\
& E\left[\omega_{v 0}^{l} \cdot \omega_{v 1}^{l}\right]=0
\end{aligned}
$$

As the noise of $v_{s l}^{r}$ is $\omega_{v 0}^{l}-\omega_{v 1}^{l}$, the noise variance of $v_{s l}^{r}$ is

$$
E\left[\left(\omega_{v 0}^{l}-\omega_{v 1}^{l}\right)^{2}\right]=E\left[\left(\omega_{v 0}^{l}\right)^{2}\right]-2 E\left[\omega_{v 0}^{l} \cdot \omega_{v 1}^{l}\right]+E\left[\left(\omega_{v 1}^{l}\right)^{2}\right]=2 R_{v}
$$

From above equations, we can see that the measurement noise increases. However, the velocity measurement noise caused by the spectrometer is $1 \mathrm{~cm} / \mathrm{s}$, while the predicted radial velocity error of star is on the order of $10 \mathrm{~m} / \mathrm{s}$. Therefore, it is worthy to eliminate the predicted radial velocity error of star at the cost of the increase of measurement noise.

(3) The application situations. This relative navigation is fit for the whole space since the starlight can be observed in the whole space. As we know, in the vision-based relative navigation, the observed spacecraft may be rarely visible to the observer's star sensor in the case that the spacecrafts are far from each other[10]. Unlike the vision-based relative navigation, the starlight frequency shift-based relative navigation method does not observe the spacecraft reflectivity. As this relative navigation method observes the starlight, it can work very well even if the distance between two spacecrafts is very long.

Moreover, like the inter-satellite links, the relative velocity measurement method based on starlight can be applied to several spacecrafts.

\section{Navigation Filter}

As the navigation system for the Mars explorer formation flight is nonlinear, we employ the EKF as the navigation filter due to its good performance in nonlinear estimation. The equations for extended Kalman filter can be found in Reference[12].

In order to make the celestial autonomous navigation system observable, we integrate the relative velocity measurement with the X-ray pulsar navigation[13] and the inter-satellite links, and propose the velocity measurement/pulsar/inter-satellite links integrated navigation. 
185

186

In the process of the pulsar observation, only the relative velocity measurement and the intersatellite links can be obtained. The measurement model and measurement value can be expressed as:

$$
\begin{aligned}
h(\boldsymbol{X}, t) & =\left[\begin{array}{l}
h^{s}(\boldsymbol{X}, t) \\
h^{I}(\boldsymbol{X}, t)
\end{array}\right] \\
\boldsymbol{Y} & =\left[\begin{array}{l}
\boldsymbol{Y}^{s} \\
\boldsymbol{Y}^{I}
\end{array}\right]
\end{aligned}
$$

Once the pulse TOA is obtained, three measurements can be utilized. The measurement model and the measurement value can be represented as:

$$
\begin{aligned}
h(\boldsymbol{X}, t) & =\left[\begin{array}{l}
h^{s}(\boldsymbol{X}, t) \\
h^{I}(\boldsymbol{X}, t) \\
h^{X}(\boldsymbol{X}, t)
\end{array}\right] \\
\boldsymbol{Y} & =\left[\begin{array}{l}
\boldsymbol{Y}^{s} \\
\boldsymbol{Y}^{I} \\
\boldsymbol{Y}^{X}
\end{array}\right]
\end{aligned}
$$

\section{Simulation Results}

As the relative velocity navigation system based on starlight is unobservable and can only be utilized as an aided navigation, it is hard to investigate its navigation performance. In order to demonstrate the feasibility and effectiveness of the relative velocity measurement method, we combine it with the $\mathrm{X}$-ray pulsar navigation, and propose the integrated navigation system with observability in this paper. In this section, we compare the integrated navigation with the X-ray pulsar navigation, and show the simulation results in this section. It is worth to be mentioned that the pulsar navigation in this paper employs the inter-satellite links besides the pulse TOA. The simulation conditions are shown as follows.

We refer the orbit of American Mars Pathfinder, and design the simulation orbit for the Mars explorer formation flight. The initial orbit elements of two Mars explorers in formation flight are shown in Table 1. The simulation time is from 1 Mar 1997 00:00:00.00 UT to 2 Mar 1997 00:00:00.00 UT. The orbits of Mars explorers are simulated with the Satellite Tool Kit (STK), which takes the perturbation effects into account.

The creation of a catalog of pulsars is most useful for deep space navigation. The figure of merit for X-ray pulsar assists in identifying pulsars with the potential to provide good timing and range accuracy, and its expression can be found in reference[13]. It is assumed that there is no clock error for the spacecraft. Thus, in order to make the navigation system observable, three X-ray pulsars are required. In this paper, three commonly used pulsars, whose figures of merit are highest in all pulsars, are adopted, and the right ascension angles and declination angles of those pulsars are shown as Table 2. The pulse TOA measurement accuracy can be calculated as reference[13]. The X-ray background radiation flux is $0.005 \mathrm{ph} / \mathrm{cm} 2 / \mathrm{s}$. The area of the X-ray sensor is $1 \mathrm{~m} 2$. The X-ray pulsar observation period is $1000 \mathrm{~s}$.

Two brightest stars (Sirius, Canopus) are adopted as the navigation stars. Their parameters are shown in Table 3. 
Table 1. Initial orbital elements of the American Mars Pathfinder

\begin{tabular}{ccc}
\hline Orbital element & Mars explorer 0 & Mars explorer 1 \\
\hline Semimajor Axis & $193216365.381 \mathrm{~km}$ & $193216365.381 \mathrm{~km}$ \\
Eccentricity & 0.236386 & 0.236386 \\
Inclination & $23.455^{\circ}$ & $23.455^{\circ}$ \\
Right Ascension of Ascending & $0.258^{\circ}$ & $0.258^{\circ}$ \\
Node & $71.347^{\circ}$ & $71.347^{\circ}$ \\
Argument of Periapsis & $85.152^{\circ}$ & $85.153^{\circ}$ \\
True Anomaly & & \\
\hline
\end{tabular}

216

Table 2. Parameters of pulsars

\begin{tabular}{cccc}
\hline Pulsar & B0531+21 & B1821-24 & B1937+21 \\
Right ascension angle/ $^{\circ}$ & 83.63 & 276.13 & 294.92 \\
$\begin{array}{c}\text { Declination angle/ } \\
\text { Measurement }\end{array}$ & 22.01 & -24.87 & 21.58 \\
$\begin{array}{c}\text { accuracy/m } \\
\text { Men }\end{array}$ & 77.75 & 202.09 & 192.04 \\
\hline
\end{tabular}

Table 3. Parameters of stars

\begin{tabular}{|c|c|c|}
\hline Star & Sirius & Canopus \\
\hline Right ascension angle/ ${ }^{\circ}$ & 101.29 & 95.99 \\
\hline Declination angle/ ${ }^{\circ}$ & -16.72 & -52.70 \\
\hline
\end{tabular}

Table 4. Parameters of navigation filter

\begin{tabular}{lc}
\hline Parameter & Value \\
\hline Number of X-ray sensors & 3 \\
Number of spectrometers & 2 \\
The measurement error of \\
$\quad \begin{array}{l}\text { spectrometer } \\
\text { The predicted radial velocity } \\
\quad \text { error of star }\end{array}$ & $0.01 \mathrm{~m} / \mathrm{s}$ \\
$\begin{array}{l}\text { The error of inter-satellite links } \\
\text { The X-ray pulsar observation } \\
\text { period }\end{array}$ & $10 \mathrm{~m} / \mathrm{s}$ \\
The star observation period & $1000 \mathrm{~s}$ \\
\hline
\end{tabular}


The inter-satellite links observation period

Initial state errors

$$
\delta \boldsymbol{X}^{0}(0)=[5200 m, 5200 m, 5200 m, 19 m / s, 19 m / s, 14 m / s]
$$

$$
\delta \boldsymbol{X}^{(1)}(0)=[6000 m, 6000 m, 6000 m, 20 m / s, 20 m / s, 15 m / s]
$$

Initial estimation error covariance

$\boldsymbol{P}(0)$ is selected at random

State process noise covariance

$$
\boldsymbol{Q}=\operatorname{diag}\left[q_{1}^{2}, q_{1}^{2}, q_{1}^{2}, q_{2}^{2}, q_{2}^{2}, q_{2}^{2}\right], q_{1}=2 \mathrm{~m} q_{2}=3 \times 10^{-3} \mathrm{~m} / \mathrm{s}
$$

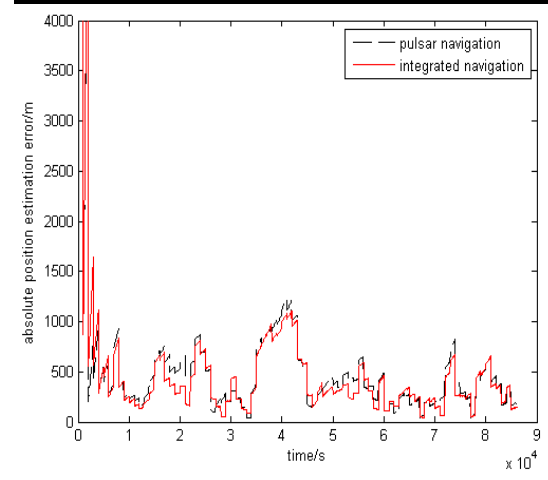

(a) absolute position error

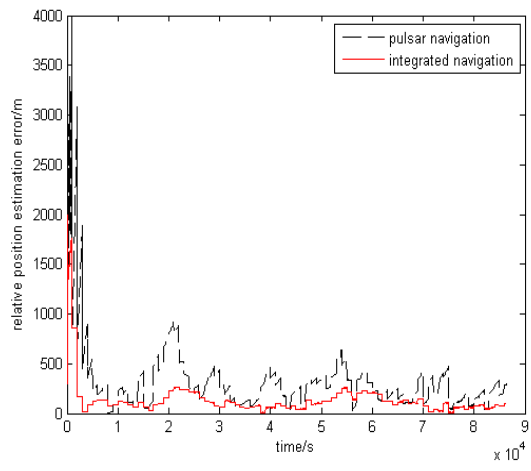

(c) relative position error

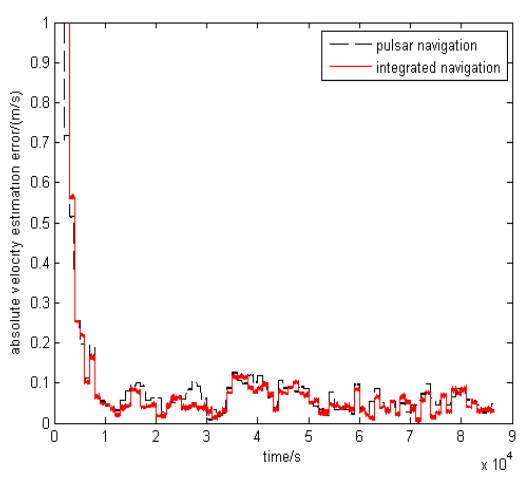

(b) absolute velocity error

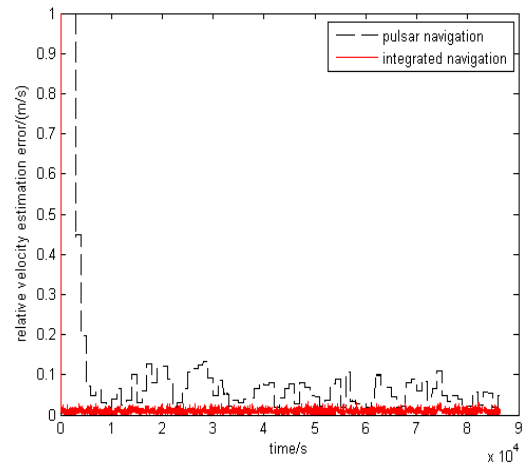

(d) relative velocity error

Figure.2. The estimation errors for pulsar navigation and integrated navigation

The other parameters of the navigation filter are shown as Table 4.

We investigate the performance of the integrated navigation and the pulsar navigation. Figure 2 shows the performance comparison of these two navigation methods using the same simulation conditions described above. As the results in Figure 2 demonstrate, the two methods can converge very well, and the integrated navigation performs considerably better. Table 5 shows the comparison between the integrated navigation and the $\mathrm{X}$-ray pulsar navigation over $100 \mathrm{Montè-Carlo} \mathrm{trials.} \mathrm{It} \mathrm{can}$ be seen that compared with the pulsar navigation, the integrated navigation shows $7.4 \%$ and $17.0 \%$ improvement for absolute position and velocity, $63.9 \%$ and $85.1 \%$ for relative position and velocity, respectively. From these results, we can see that the integrated navigation improves the navigation performance obviously, especially the relative navigation. The reason is that the starlight frequency shift-based relative navigation method provides the highly accurate relative velocity information. From above simulation results, we can see that the relative velocity navigation based on starlight is an effective aided navigation method. 
Table 5. Comparision of two navigation mehods.

\begin{tabular}{ccccc}
\hline $\begin{array}{c}\text { Navigation } \\
\text { method }\end{array}$ & $\begin{array}{c}\text { Absolute } \\
\text { position }\end{array}$ & $\begin{array}{c}\text { Absolute } \\
\text { velocity }\end{array}$ & $\begin{array}{c}\text { Relative } \\
\text { position }\end{array}$ & $\begin{array}{c}\text { Relative } \\
\text { velocity }\end{array}$ \\
\hline Pulsar navigation & $359.85 \mathrm{~m}$ & $0.0576 \mathrm{~m} / \mathrm{s}$ & $273.81 \mathrm{~m}$ & $0.0642 \mathrm{~m} / \mathrm{s}$ \\
$\begin{array}{l}\text { Integrated } \\
\text { navigation }\end{array}$ & $333.23 \mathrm{~m}$ & $0.0478 \mathrm{~m} / \mathrm{s}$ & $98.74 \mathrm{~m}$ & $0.0096 \mathrm{~m} / \mathrm{s}$ \\
\hline
\end{tabular}

Next, we investigate the impact of the X-ray sensor's area on both the integrated navigation and the X-ray pulsar navigation. Figure 3 represents the simulation results with different area of sensor over 50 Montè-Carlo trials. We can see that the performance of the two methods improves with enlarging the area of X-ray sensor, and the integrated navigation method outperforms $\mathrm{X}$-ray pulsar navigation with the same area, especially the relative navigation.

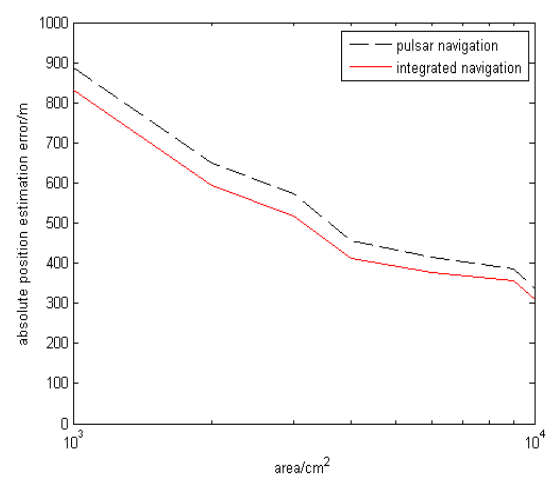

(a) absolute position error

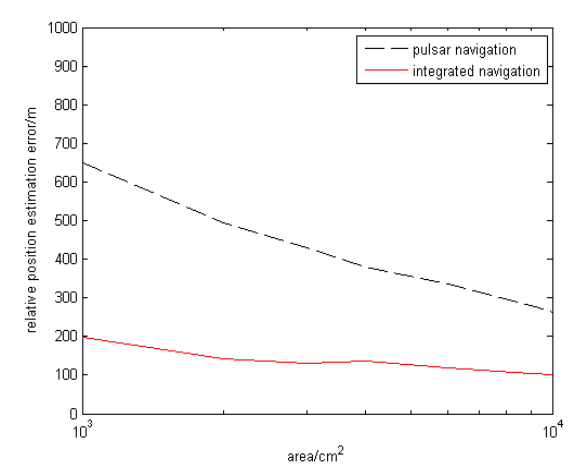

(c) relative position error

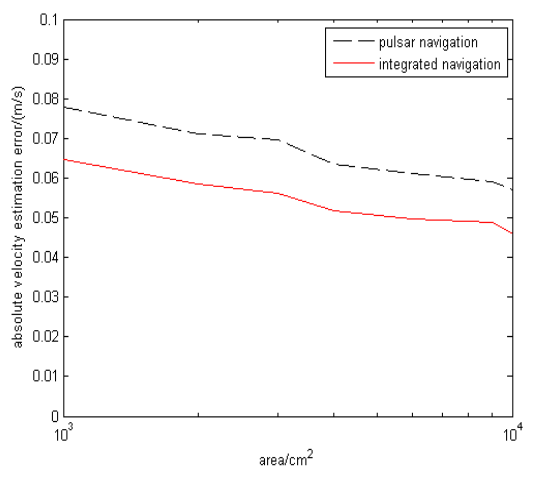

(b) absolute velocity error

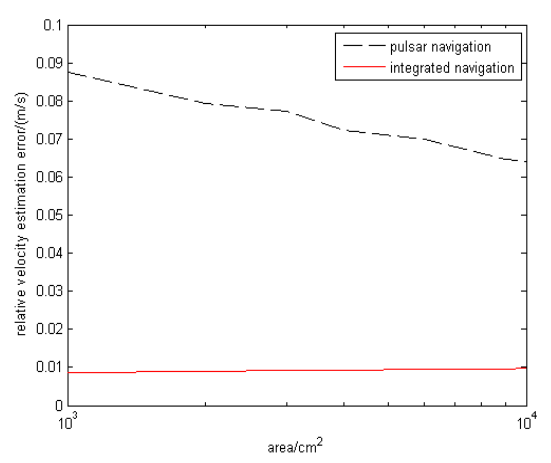

(d) relative velocity error

Figure.3. The estimation errors with different area of X-ray sensor 


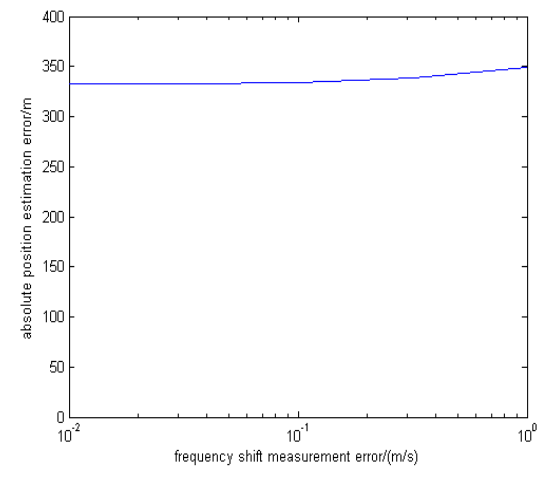

(a) absolute position error

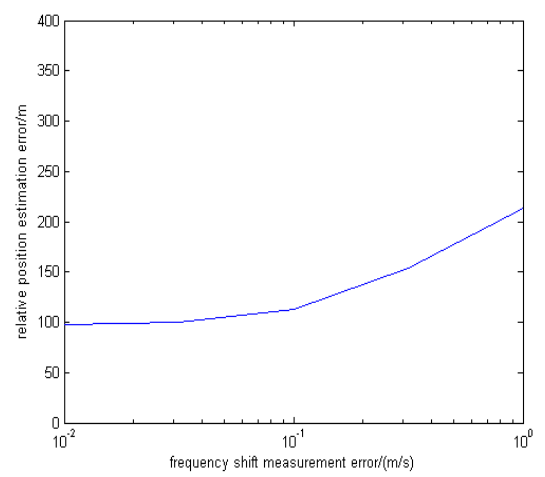

(c) relative position error

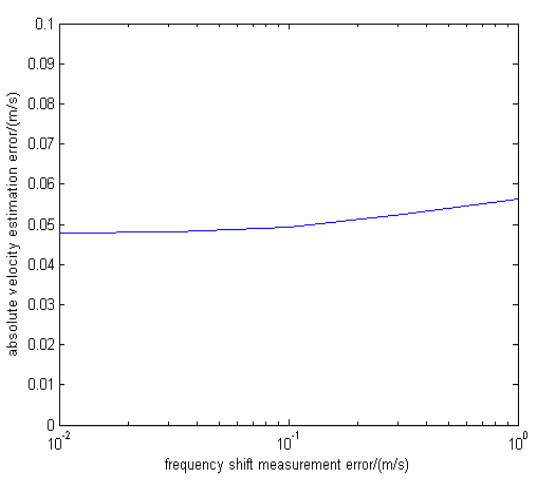

(b) absolute velocity error

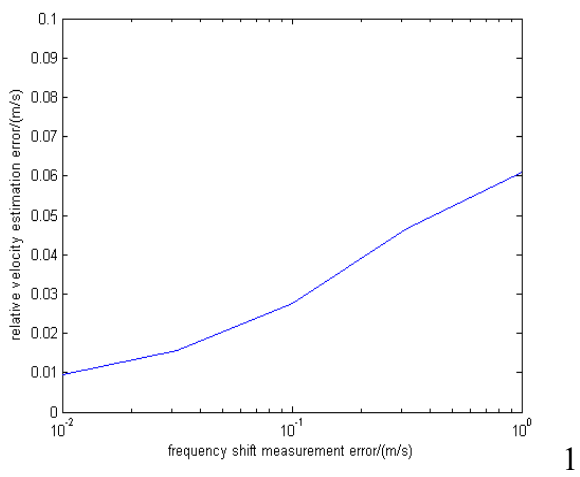

(d) relative velocity error

Figure.4. The estimation accuracy with different frequency shift measurement accuracy

Finally, we investigate the impact of the starlight's frequency shift measurement accuracy on the integrated navigation. Figure 4 represents the simulation results with different accuracy of the frequency shift measurement over 50 Montè-Carlo trials. We can see that both absolute and relative navigation performance improves with the decline of the starlight's frequency shift measurement error, especially the relative navigation. From Figure 4 and Table 5, it is interesting to note that the integrated navigation accuracies with different frequency shift measurement error are higher than that of X-ray pulsar navigation.

\section{Conclusions}

In this paper, a novel relative velocity measurement method is developed, which employs the spectrometers as the sensors and gets the relative velocity between a pair of Mars explorers from the starlight. It can be only utilized as an aided navigation method due to its unobservability. We combine it with X-ray pulsar navigation, and propose the starlight Doppler/pulsar integrated navigation method for formation flight. In the proposed integrated navigation system, the relative velocity, the pulse TOA and the inter-satellite link are the navigation measurements. The simulation results demonstrate that both absolute and relative autonomous navigation accuracy of the proposed integrated navigation method are higher than that of the X-ray pulsar navigation, especially the relative navigation. And the proposed method improves the velocity estimation accuracy greatly, especially the relative one.

The relative velocity measurement method for formation flight has the following virtues: (1) it is completely autonomous; (2) it can work in whole space; (3) it provides highly-accurate relative velocity; (4) it is not subjected to the distance between a pair of Mars explorers. Therefore, this relative velocity measurement method is a good aided navigation method for formation flight. Besides the advantages of the relative velocity measurement method, the proposed integrated navigation can 
also provide position information. Thus, this integrated navigation system is a good choice for the Mars explorer formation flight.

Acknowledgments: This work was supported by the National Natural Science Foundation of China under grant No. 61501336, the Natural Science Foundation of Jiangxi Province of China under Grant No. 20161BAB202037, the Science and Technology Research Project of Jiangxi Province Education Department of China under Grant No. GJJ160696 and the Jiangxi Province Key Laboratory of Water Information Cooperative Sensing and Intelligent Processing of China under Grant No. 2016WICSIP007.

Author Contributions: Zhonghua Wang and Jiu Liu conceived and designed the experiments; Yiming Peng performed the experiments; Zhonghua Wang and Yiming Peng analyzed the data; Jiu Liu contributed materials and analysis tools; Zhonghua Wang wrote the paper.

Conflicts of Interest: The authors declare no conflict of interest.

\section{References}

1. A. Mehrabian; K. Khorasani. Distributed and cooperative quaternion-based attitude synchronization and tracking control for a network of heterogeneous spacecraft formation flying mission. Journal of the Franklin Institute 2015, vol. 352, no. 9, pp. 3885-3913, DOI: 10.1016/j.jfranklin.2015.04.007.

2. D. P. Scharf, S, R, Martin, C. C. Liebe, Precision formation flying at megameter separations for exoplanet characterization. Acta Astronautica 2016,vol. 123, pp. 420-434, DOI: 10.1016/j.actaastro.2015.12.044 .

3. J. Y. Wu; J. C. Fang, and Z. H. Yang. ASUKF based relative navigation method for mars probe using pulsar. Chinese Journal of Scientific Instrument 2013, vol. 34, no. 8, pp. 1711-1716, DOI: 10.3969/j.issn.02543087.2013.08.005.

4. X. Ning; M. Gui and J. Fang. A Novel Differential Doppler Measurement-Aided Autonomous Celestial Navigation Method for Spacecraft During Approach Phase. IEEE Transactions on Aerospace and Electronic Systems 2017, vol. 53, no. 2, pp. 587-597, DOI: 10.1109/taes.2017.2651558.

5. Ma X; Fang J and Ning X. Autonomous celestial navigation for a deep space probe approaching a target planet based on ephemeris correction. Part G: Journal of Aerospace Engineering 2015, vol. 229, no. 14, pp. 2681-2699, DOI: 10.1177/0954410015586841.

6. P. J. Buist; S. Engelen and A. Noroozi. Overview of Pulsar Navigation: Past, Present and Future Trends. Navigation 2011, vol. 58, no. 2, pp. 153-164, DOI: 10.1002/j.2161-4296.2011.tb01798.x .

7. J. Liu; J. Wu; L. Xiong; J. C. Fang and L. Guang. Fast position and velocity determination for pulsar navigation using NML and LSM. Chinese Journal of Electronics 2017, vol. 26, no. 6, pp. 1325-1329, DOI: 10.1049/cje.2017.09.005.

8. Y. d. Wang; W. Zheng and S. M. Sun. Pulsar profile denoising using kernel regression based on maximum correntropy criterion. Optik-International Journal for Light and Electron Optics 2017, vol. 130, pp. 757-764, DOI: 10.1016/j.ijleo.2016.10.132.

9. A. A. Emadzadeh; J. L. Speyer. Relative navigation between two spacecraft using X-ray pulsars. IEEE Transactions on Control Systems Technology 2011, vol. 19, no. 5, pp. 1021-1035, DOI: 10.1109/tcst.2010.2068049.

10. K. Xiong; C. Wei and L. Liu. Autonomous navigation for a group of satellites with star sensors and intersatellite links. Acta Astronautica 2013, vol. 86, no.3, pp. 10-23, DOI: 10.1016/j.actaastro.2012.12.001.

11. K. Xiong; C. L. Wei and L. D. Liu. The use of X-ray pulsars for aiding navigation of satellites in constellations. Acta Astronautica 2009, vol. 64, no. 4, pp. 427-436, DOI: 10.1016/j.actaastro.2008.09.005.

12. J. C. Fang; X. L. Ning and J. Liu. Principles and Methods of spacecraft celestial Navigation, 2nd Edition; National Defense Industry Press: Beijing, China, 2017; ISBN: 9787118043273. 
307 13. S. I. Sheikh; D. J. Pines and P. S. Ray. Spacecraft navigation using x-ray pulsars. Journal of Guidance and 308 Control and Dynamics 2006, vol. 29, no. 1, pp. 49-63, DOI: 10.2514/1.13331. 